\title{
LA POLÍTICA DE LA ADMINISTRACIÓN TRUMP PARA AMÉRICA LATINA: ¿REINVENCIÓN DE LA DOCTRINA MONROE?
}

\author{
Luis Fernando Ayerbe ${ }^{1}$
}

\begin{abstract}
Durante la administración de Barack Obama, Estados Unidos alcanzaron una situación regional políticamente confortable, con mayoría de gobiernos aliados, especialmente de las cuatro principales economías, Brasil, México, Argentina y Colombia. Reforzando ese contexto, se profundiza la pérdida de influencia del bolivarianismo, a partir de la crisis económica enfrentada por el gobierno de Nicolás Maduro, y se normalizan las relaciones diplomáticas con Cuba. Con el ascenso de Donald Trump, la lectura de ese escenario sufre alteraciones, con la percepción de un momento favorable a la retomada de un hegemonismo intervencionista plasmado en la tradición de la Doctrina Monroe, en que la perspectiva de coexistencia con la pluralidad de formas de gobierno tiende a ser substituida por una apuesta en la eliminación del campo adversario.
\end{abstract}

Palabras clave: Donald Trump; América Latina; hegemonía; monroísmo.

\section{A POLÍTICA DO GOVERNO TRUMP PARA A AMÉRICA LATINA: REINVENÇÃO DA DOUTRINA MONROE?}

Durante a administração de Barack Obama, os Estados Unidos alcançaram uma situação regional politicamente confortável, com maioria de governos aliados, especialmente das quatro principais economias, Brasil, México, Argentina e Colômbia. Reforçando esse contexto, se aprofunda a perda de influência do bolivarianismo, a partir da crise econômica enfrentada pelo governo de Nicolás Maduro, e se normalizam as relações diplomáticas com Cuba. Com a ascensão de Donald Trump, a leitura desse cenário sofre alterações, com a percepção de um momento favorável a retomada de um hegemonismo intervencionista plasmado na tradição da Doutrina Monroe, em que a perspectiva de coexistência com a pluralidade de formas de governo tende a ser substituída por uma aposta na eliminação do campo adversário.

Palavras-chave: Donald Trump; América Latina; hegemonia; monroísmo.

\section{THE POLICY OF THE TRUMP ADMINISTRATION FOR LATIN AMERICA: REINVENTION OF THE MONROE DOCTRINE?}

During the administration of Barack Obama, the United States reached a politically comfortable regional situation, with most allied governments, especially the four major economies, Brazil, Mexico, Argentina and Colombia. Reinforcing this context deepens the loss of influence of Bolivarianism, as a result of the economic crisis faced by the government of Nicolás Maduro, and normalizes diplomatic relations with Cuba. With the rise of Donald Trump, the reading of this scenario changes, with the perception of a favorable moment to the resumption of interventionist hegemonism embodied in the tradition of the Monroe Doctrine, in which the perspective of

\footnotetext{
1. Profesor titular de historia general de la Universidade Estadual Paulista (Unesp), actuando en el Departamento de Economía de la Facultad de Ciencias y Letras de Araraquara y en el programa San Tiago Dantas de posgrado en relaciones internacionales de la Unesp, Universidade Estadual de Campinas (Unicamp) y Pontifícia Universidade Católica de São Paulo (PUC-SP).E-mail: <luisfernandoayerbe@gmail.com>.
} 
coexistence with the plurality of forms of government tends to be replaced by a bet on the elimination of the opponent's field.

Keywords: Donald Trump; Latin America; hegemony; monroism.

JEL: F51.

\section{INTRODUCCIÓN}

En contraste con Barack Obama, cuya política hemisférica apostó en una construcción de hegemonía pautada en la distensión con gobiernos situados en campo no alineado, especialmente Cuba, Donald Trump esboza una retomada de la lógica intervencionista plasmada en la tradición de la Doctrina Monroe, en que la perspectiva de coexistencia con la pluralidad de formas de gobierno tiende a ser substituida por una apuesta en la eliminación del campo adversario.

El fenómeno Donald Trump, inicialmente subestimado en el proceso electoral de 2016 como expresión del voluntarismo de empresario narcisista y con discurso anti-establishment movido menos por convicción que por oportunismo, termina imponiéndose como catalizador de un cambio de perspectiva sobre la inserción de Estados Unidos en el mundo. America First será el slogan de nuevo credo patriótico que pretende restituir una grandeza que se considera sacrificada en nombre de supuestas responsabilidades globales cuyo costo-beneficio habría sido negativo para el país. Inmigración, proteccionismo, libre-comercio, multilateralismo, seguridad internacional, compromisos con aliados, son temas de destaque de una pauta sobre las relaciones con el exterior impuesta por Trump a elites establecidas que, en su desconcierto, y más allá de simpatías partidarias, terminan aglutinándose en torno de la candidatura demócrata de Hillary Clinton.

El favoritismo atribuido a Clinton por parte de encuestas y analistas, fortalecido con apoyo mayoritario de formadores de opinión del ámbito empresarial, sindical, artístico y medios de comunicación, duró hasta la apertura de las urnas, cuando el escrutinio de votos pasó a mostrar ventaja continua de su oponente en la conquista de delegados para el colegio electoral que lo sacramentó como presidente.

Ya desde los primeros días de gobierno, inició ofensiva en la implementación de su programa. Entre los ejemplos a destacar en el ámbito internacional, órdenes ejecutivas decretando la salida del Acuerdo Transpacífico de Cooperación Económica (TPP), iniciativa negociada por Obama, y la construcción de muro en la frontera con México; palabras elogiosas al resultado del Brexit que aprobó la salida de Gran Bretaña de la Unión Europea; la proposición de Ted Malloch como representante de Estados Unidos en la organización, que en declaraciones anteriores a la $\mathrm{BBC}$ había previsto el fin del euro en un año y medio, generando el rechazo de su nombre por parte de la Eurocámara; la salida del Acuerdo de 
Paris sobre cambio climático; el rompimiento unilateral del acuerdo nuclear con Irán retomando las sanciones al país; medidas proteccionistas estableciendo tarifas a la importación de acero y aluminio, afectando aliados como Canadá y la Unión Europea; y despliegue de ofensiva contra China a través de la imposición de restricciones a la importación de productos objetivando forzar una balanza comercial más favorable al país.

En términos estructurales, las prioridades pautadas en la noción de America First están presentes en los presupuestos propuestos al Congreso para los años fiscales de 2018 y 2019, en que se visualiza claramente el énfasis en seguridad y defensa en detrimento de la diplomacia. Para el Departamento de Defensa, se propone un aumento de $10 \%$ para 2018 y de $17 \%$ para 2019. Para el Departamento de Seguridad Interna, aumento de $7 \%$ y $8 \%$ respectivamente, direccionado principalmente para la frontera con México, envolviendo la construcción del muro y gastos de infraestructura y personal asociados al control migratorio. Para el Departamento de Estado, cortes de 28\% en 2018 y de 26\% en 2019, con reducción de recursos para Asistencia al Desarrollo (DA), con impacto en la actuación de la Organización de las Naciones Unidas (ONU), que también es afectada en programas contra el cambio climático, como parte de los recortes en la Agencia de Protección Ambiental, correspondientes a 31\% en 2018 y 34\% en 2019 (United States of America, 2017; 2018d).

Cabe considerar que se trata de solicitación presupuestaria presentada al Congreso, en que Trump viene enfrentando dificultades para viabilizar el financiamiento de sus prioridades. En febrero de 2018, cuando fue presentado el presupuesto de 2019, aún no había sido aprobado el del año anterior, siendo que el resultado final puede ser diferente al propuesto por el Poder Executivo, como ejemplifica el financiamiento del muro fronterizo con México, bloqueado por la Cámara de Diputados, donde el Partido Demócrata conquisto la mayoría en las elecciones de noviembre de 2018.

\section{RELACIONES CON AMÉRICA LATINA: ¿TIEMPOS DE DOCTRINA MONROE?}

Sin haber presentado durante las elecciones de 2016 cualquier lineamiento de alcance regional, se limitó a propuestas en temas puntuales que esbozan un cambio de posiciones en la política hemisférica en dos frentes: i) las relaciones con los vecinos norteamericanos en cuestiones de inmigración y libre-comercio, en que se destacan la antes mencionada construcción de muro fronterizo con México y la revisión del Tratado de Libre-Comercio de América del Norte (TLCAN), en vigor desde la administración Clinton, que a partir de proceso negociador es refundado en octubre de 2018 bajo la denominación de Acuerdo Estados Unidos-México-Canadá (USMCA); y ii) la injerencia más abierta por el cambio de gobierno en países situados en campo no-alineado, como Cuba, Venezuela y Nicaragua. 
En ese segundo tema, el proceso de endurecimiento no responde a un planeamiento estratégico inicial, más al ajuste entre capacidad de adaptación de los funcionarios responsables por las relaciones internacionales del país y la improvisación impulsiva que marca el estilo presidencial, conllevando a una rotación de visiones diferentes en el comando de la política exterior.

Durante la gestión de Rex Tillerson como secretario de Estado, no habrá novedades importantes de contenido en la política hemisférica, prevaleciendo el pragmatismo sobre la ideología. Antes de llegar al gobierno, Tillerson se desempeñó como chief executive officer (CEO) de la multinacional petrolera Exxon Mobil, en que adquirió reconocimiento por su habilidad negociadora en defensa de intereses de la empresa frente a obstáculos asociados a la política externa estadounidense, como las sanciones de Obama a Rusia en respuesta a la interferencia en Ucrania, siendo homenajeado en 2013 por el presidente Vladimir Putin con el Orden de la Amistad rusa.

Teniendo que lidiar con las presiones de liderazgos conservadores frente a la promesa de campaña de Trump de revisión de los acuerdos firmados por su antecesor con el gobierno cubano en el marco de la normalización de relaciones diplomáticas, el tono de la respuesta combinará palabras duras con anuncio de medidas de escaso impacto real. Conforme expresa el discurso presidencial de 16 de junio de 2017 en Miami:

no levantaremos las sanciones contra el régimen cubano hasta que todos los prisioneros políticos sean liberados, las libertades de reunión y expresión sean respetadas, sean legalizados todos los partidos políticos y se programen elecciones libres y supervisadas internacionalmente (...) Vamos a restringir muy fuertemente los dólares americanos que fluyen para los servicios militares, de seguridad y de inteligencia que son el núcleo del régimen de Castro (...) Vamos a hacer cumplir la prohibición del turismo. Vamos a hacer cumplir el embargo. Tomaremos medidas concretas para garantizar que las inversiones fluyan directamente para las personas, para que ellas puedan abrir empresas privadas y comenzar a construir el excelente futuro de su país (Theater, 2017, traducción nuestra).

El discurso apunta para un ajuste de medidas que, además de ambiguo en su alcance efectivo, es de aplicabilidad controversial, ya que la dinámica alcanzada desde el inicio de la normalización diplomática, principalmente en el área del turismo, con creciente flujo de ciudadanos e inversiones de capitales estadounidenses, coloca sectores empresariales y buena parte de la opinión pública del país en camino opuesto del retroceso anunciado.

Recién en febrero de 2018, poco más de un año desde la Asunción de Trump a la Casa Blanca, Tillerson realizará una visita a países de América Latina, específicamente México, Argentina, Perú, Colombia y Jamaica. Además de buscar 
coincidencias para una profundización de sanciones contra el gobierno de Nicolás Maduro en Venezuela, el principal registro de la visita será la revalorización del monroísmo como pauta de orientación de las relaciones con la región.

Respondiendo a pregunta del público al final de discurso en la Universidad de Austin en que anuncia el viaje a la región, cuestionado sobre como visualiza la trayectoria de la Doctrina Monroe en términos de suceso o fracaso, Tillerson asume que "fue un compromiso importante en la época, y pienso que a lo largo de los años continuó como marco de la relación (...) Entonces creo que es tan relevante hoy como lo fue en el día en que se ha escrito" (United States of America, 2018a).

Si bien su declaración no tiene efectos prácticos inmediatos, ya que Tillerson deja el cargo em marzo, substituido por Mike Pompeo, director de la Central Intelligence Agency (CIA) y miembro del Tea Party, su invocación de la doctrina termina siendo el punto inaugural de la visión estratégica de las relaciones hemisféricas ensayada por la administración Trump, estableciendo un divisor de aguas con relación a su antecesor en la Casa Blanca.

De hecho, en noviembre de 2013, John Kerry, secretario de Estado de Barack Obama en su segundo mandato, había anunciado en discurso en la Organización de los Estados Americanos (OEA) que "la era de la Doctrina Monroe terminó", apuntando para nueva realidad en que ya no se "precisa de fuerza para tener fuerza" (Kerry, 2013).

Presentada en 1823, en contexto de preocupación con las intenciones de España de revertir, con el apoyo de la Santa Alianza, el proceso de independencia latinoamericano, la Doctrina Monroe establece límites a la intervención de potencias europeas en el continente. Aunque nunca haya sido formalmente abandonada hasta el discurso de Kerry, su invocación explícita va hasta 1904, cuando es formulado por Theodore Roosevelt su quinto corolario (Ayerbe, 2002). No obstante, terminó siendo asociada con el papel de guardián hemisférico que Estados Unidos se atribuye, justificando el intervencionismo a lo largo de la historia a partir de tres argumentos principales: i) la existencia de ambiciones expansionistas en la región por parte de potencias extracontinentales; ii) la defensa del denominado "modo de vida americano", que se pretende expresión del mayor grado de avance conocido por la civilización; y iii) la fragilidad de las naciones latinoamericanas para defender sus propios intereses sin la ayuda del gran vecino del norte.

La declaración de Kerry corresponde a una percepción favorable del contexto hemisférico, cuando estaban en curso las negociaciones secretas con Cuba que llevarían al anuncio de la normalización diplomática en diciembre de 2014. En la evaluación de Obama, ese avance removería el principal obstáculo a la neutralización del antiamericanismo que prevalecía en el momento de su llegada 
al gobierno, en que la influencia del bolivarianismo componía parte destacada de la agenda regional (Ayerbe, 2016).

De hecho, la evolución posterior contribuyó para fortalecer la perspectiva de la administración Obama, con el debilitamiento de la influencia regional bolivariana a partir de la crisis económica enfrentada por el gobierno de Nicolás Maduro paralelamente a la victoria electoral de la oposición en las elecciones legislativas de 2015, obteniendo mayoría en la Asamblea Nacional; el impacto de los cambios de gobierno en Argentina y Brasil con la elección de Mauricio Macri y el impeachment de Dilma Rousseff, cuando Venezuela pierde dos aliados fundamentales.

La reversión por parte del gobierno Trump de la postura asumida por Obama adquiere relevo a partir de marzo de 2018, con cambio de equipo en la política exterior, en que paralelamente al nombramiento de Mike Pompeo en el Departamento de Estado, John Bolton - subsecretario de Estado para el Control de Armas y Seguridad Internacional, y representante en la ONU en la administración de George W. Bush - asume el cargo de asesor de Seguridad Nacional. A partir de este momento, se tornan explícitas las intenciones de retomada del activismo del país en la región, buscando llevar a la práctica la invocación del monroísmo inaugurada por Tillerson. Conforme expresó Bolton (2018) meses antes de ser llamado para la Casa Blanca:

con Venezuela en las cuerdas, la legitimidad revolucionaria de los Castros desaparece, y con la presión de Estados Unidos aumentando, por cuanto tiempo el régimen sobrevive es un tema en abierto (...) Aunque las tensiones probablemente no retornen a los niveles de la Guerra Fría, cuando la crisis soviética sobre Cuba llegó cerca de provocar la guerra nuclear, la intromisión rusa en América Latina podría inspirar Trump a reafirmar la Doctrina Monroe (otra víctima de los años Obama) y erguirse para el pueblo sitiado de Cuba (como él hace ahora con Irán) (op. cit., 2018, traducción nuestra).

La cita de Bolton (2018) explicita las tres dimensiones apuntadas anteriormente cuando nos referimos a los pilares del mon roísmo como justificativa de la intervención en la región: i) la percepción de amenaza de potencias extrarregionales, en este caso Rusia, a la que se agregará posteriormente China; ii) la defensa del modo de vida estadounidense, ejemplificado en la promoción de cambios de gobierno en Venezuela y Cuba teniendo como referencia el modelo de democracia vigente en el país; y iii) la supuesta situación indefensa en que se encontrarían los pueblos en esos países para ejercer su ciudadanía sin el apoyo de Estados Unidos.

El cambio de política frente a la administración anterior con relación a gobiernos situados en campo adversario adquiere perfil más explícito en dos 
dimensiones: i) mayor injerencia en Nicaragua, Cuba y Venezuela; y ii) estado de alerta con la presencia militar de Rusia, principalmente en Venezuela, y la expansión económica de China a escala regional.

En la primera dimensión, la crisis política interna de Nicaragua abre espacio para una redefinición bien explicita. En noviembre de 2016, Daniel Ortega obtuvo su tercer mandato consecutivo al vencer las elecciones con más de $70 \%$ de los votos. Tanto en el frente interno como externo, incluyendo Estados Unidos, Nicaragua era percibida en situación de estabilidad política, y su gobierno asumido como parte legítima del status quo. En abril de 2018, respondiendo a preocupaciones del Fondo Monetario Internacional (FMI) sobre la sostenibilidad del sistema se seguridad social, se anuncia a través de decreto una reforma que aumenta las contribuciones de empresas y trabajadores, y reduce en 5\% las jubilaciones. La medida es respondida por manifestaciones en las calles, lideradas por sectores estudiantiles y fuertemente reprimidas, resultando en al menos tres muertes, dos manifestantes y un policía, y decenas de heridos (Perry, 2018).

Frente a esos eventos, el gobierno retira la reforma previsional. Lejos de generar calma, la protesta contra una política pública específica se transforma en movimiento de oposición que reivindica como objetivos la renuncia del presidente y el llamado anticipado de elecciones. A partir de ese momento, el conflicto adquiere dimensión existencial. El gobierno busca retomar el control de las calles bajo la consigna del imperio de la ley, asociando la escalada de protestas a un golpe de Estado de grupos organizados de la oposición hermanados con sectores de la derecha estadounidense, especialmente del partido de Donald Trump. En la oposición ganan fuerza los sectores que condicionan cualquier solución a la salida de Ortega.

Por cuestiones de abordaje que orienta la escrita de este artículo, no entraremos en la discusión sobre las dinámicas internas del conflicto nicaragüense. Nuestro objetivo es analizar cambios en la política de Estados Unidos situando el contexto actual en un campo más amplio del ejercicio del poder nacional en la región.

Hasta el estallido del enfrentamiento entre gobierno y oposición no se verificaba en la política externa estadounidense un estado de alerta con relación a Nicaragua. Así eran caracterizadas las prioridades asociadas al país en el Presupuesto para Operaciones en el Exterior (POE) del Departamento de Estado para 2017, último elaborado durante la administración Obama:

Estados Unidos apoya el desarrollo de una Nicaragua en la que todos los ciudadanos se beneficien de la gobernabilidad democrática, la paz, la seguridad y el desarrollo económico. La asistencia de los Estados Unidos se enfoca en prevenir la erosión de la gobernabilidad democrática nacional, mejorar la seguridad ciudadana a lo largo de la costa caribeña de Nicaragua y en las regiones del norte, y apoyar los esfuerzos para reducir el transbordo de drogas a través de Nicaragua (United States of America, 2016, p. 462). 
Diferentemente, en las secciones de ese presupuesto dedicadas a Venezuela y Cuba, se manifiestan posiciones críticas a sus respectivos sistemas políticos. En el caso venezolano, se apunta que el poder "viene concentrándose en un único partido con un ejecutivo cada vez más autoritario" (United States of America, 2016, p. 489). En las referencias a Cuba, la posición es más dura, apuntando para "un estado autoritario que limita los derechos civiles y políticos, como el derecho de reunión pacífica, la libertad de expresión, la libertad de asociación y los derechos laborales"(op. cit., p. 406). Con relación a Nicaragua, no existen cuestionamientos a la administración de Daniel Ortega, enmarcando el país dentro de tres líneas de acción definidas como orientación para el envolvimiento en Centroamérica:

i) promover la prosperidad y la integración económica regional; ii) mejorar la seguridad mediante actividades continuas y ampliadas de la Iniciativa de Seguridad Regional de Centroamérica (CARSI); y iii) promover una mejor gobernanza (op. cit., p. 462).

El primer POE del Departamento de Estado dado a público por la administración Trump, correspondiente al año fiscal de 2018, fue atípico con relación a los anteriores, ya que no destacaba separadamente asignaciones a países, retomando ese formato en el subsecuente. En el POE para 2019, dado a conocer en 14 de marzo de 2018, Nicaragua no es contemplada en ninguna asignación específica de recursos.

La omisión de Nicaragua por parte de Trump indica que el país no entró en el radar de la política exterior, sea en términos críticos o favorables. Pocos meses después, en 30 de julio, la Casa Blanca emite comunicado en reacción a la escalada del conflicto interno con una caracterización retroactiva del gobierno Ortega que sustituye el pragmatismo realista de Obama por retórica ideologizada que asume las posiciones de la derecha más radical del Partido Republicano y de organizaciones opositoras nicaragüenses.

Después de años de elecciones fraudulentas y la manipulación del régimen de la legislación nicaragüense, así como la represión de la sociedad civil, los partidos de oposición y los medios independientes, el pueblo nicaragüense salió a la calle para pedir reformas democráticas (...) La Administración Trump ha designado a tres funcionarios nicaragüenses - Francisco Díaz, Fidel Moreno y Francisco López en virtud de la Ley Global Magnitsky por abusos contra los derechos humanos y corrupción (...). Estos son un comienzo, no un final, de sanciones potenciales (...). Estados Unidos ha anunciado una ayuda adicional de \$ 1,5 millones para continuar el apoyo a la libertad y la democracia en Nicaragua, proporcionando un salvavidas crítico para la sociedad civil, organizaciones de derechos humanos y medios independientes actualmente bajo amenaza del régimen de Ortega (traducción nuestra). ${ }^{2}$ 
El 5 de septiembre, la entonces embajadora estadounidense en la ONU, Nikki Haley, en ejercicio de funciones como presidente del Consejo de Seguridad, eleva el estado de alerta sobre Nicaragua transformando la situación política interna en cuestión de seguridad internacional, encontrando resistencias por parte de Rusia, China y Bolivia. En esa línea, el embajador ruso Vassily Nebenzia apuntó que "las diferencias deben resolverse a través de un diálogo directo y pacífico, sin presiones del exterior" (Besheer, 2018, traducción nuestra).

En dirección contraria, Haley llama el consejo a salir de la posición de observador pasivo frente a una crisis que anticipa en camino convergente al de Venezuela y Siria.

El éxodo sirio ha producido millones de refugiados, sembrando inestabilidad en todo el Medio Oriente y Europa (...) El éxodo venezolano se ha convertido en el mayor desplazamiento de personas en la historia de América Latina. Un éxodo nicaragüense desbordaría a sus vecinos y crearía una oleada de migrantes y solicitantes de asilo en América Central (Besheer, 2018, traducción nuestra).

Las manifestaciones de la Casa Blanca y de la embajadora en la ONU dejan claros dos movimientos de la política exterior de Estados Unidos para generar un cambio de gobierno en Nicaragua: $i$ ) en la condena a la represión a movimientos de protesta de sectores de la sociedad, introduce como elemento novedoso la asociación de los eventos con la caracterización de la administración de Daniel Ortega como régimen, sostenido por elecciones fraudulentas, cercenamiento de libertades de la sociedad civil y de los medios independientes, situación estructural a la que se atribuye el desencadenamiento de la ida a las calles para reivindicar un proceso de democratización; y ii) paralelamente a la deslegitimación del sistema político nicaragüense, busca generar estado de alerta preventivo sobre posible desborde de la situación interna a escala regional, aprovechando para establecer un eje común de aislamiento internacional en la compañía de dos países cuyos gobernantes son situados en campo hostil, Venezuela y Siria. En la lógica de ese accionar, la prevención de amenazas a la "gobernabilidad democrática" invocada en el último presupuesto del Departamento de Estado de Obama sobre Nicaragua, cambia en tiempos de Trump para el estímulo del conflicto interno con el objetivo de anticipar la salida del presidente electo. Se transita así de una estrategia de "promover una mejor gobernanza" a la desestabilización de parámetros existentes de gestión de la sociedad. Desde esa perspectiva, se formulan sanciones dirigidas al gobierno, se amplían recursos financieros para organizaciones opositoras de la sociedad civil, y se busca instrumentalizar el Consejo de Seguridad de la ONU forzando percepciones de riesgo internacional inminente.

Aumentando la apuesta, se invoca la Ley Global Magnitsky contra funcionarios del Estado. Aprobada en 2016 por el Congreso, la ley faculta Estados 
Unidos a emitir sanciones internacionales a ciudadanos extranjeros acusados por el propio gobierno estadounidense de violaciones de derechos humanos y actos de corrupción, tornándose un eficaz instrumento de política exterior en función del peso económico del país y su capacidad de influencia en procesos de decisiones financieras a escala global.

Los cambios de marzo de 2018 en el comando de la política exterior, con destaque para el nombramiento de Bolton como asesor de Seguridad Nacional, dan coherencia a un abordaje más asertivo para la región, revalorizando un monroísmo confortable con la inducción de cambios de régimen desde el exterior.

Discursando en Miami en noviembre de 2018, con mensaje "en nombre del presidente, porque tenemos algunas preocupaciones políticas importantes a ser tratadas con relación a América Latina” (United States of America, 2018c, traducción nuestra), John Bolton oficializa la nueva postura. Por un lado, enaltece la evolución regional favorable, en que

las recientes elecciones de líderes similares en países-clave, incluyendo Ivan Duque en Colombia, y (...) Jair Bolsonaro en Brasil (...) demuestran un creciente compromiso regional con principios de libre mercado y una gobernanza abierta, transparente y responsable (op. cit., traducción nuestra).

Al mismo tiempo, incluye Cuba, Venezuela y Nicaragua en lo que denomina "troika de la tiranía", "triángulo de terror (...) causador del inmenso sufrimiento humano, el ímpetu de la enorme inestabilidad regional y la génesis de una sórdida cuna del comunismo en el hemisferio occidental" (United States of America, 2018 c, traducción nuestra). Como respuesta, proyecta una escalada de sanciones, ya que "los Estados Unidos ahora esperan ver caer cada vértice del triángulo: en La Habana, en Caracas, en Managua" (op. cit., traducción nuestra).

Se esboza en la política para América Latina una reacción de ajuste de cuentas con el pasado, una especie de revancha contra experiencias emblemáticas de la Guerra Fría, como las revoluciones cubana y sandinista, o contra aquellas que reivindican esa trayectoria, como el bolivarianismo venezolano.

Nicaragua, Cuba y Venezuela no eran situados en el campo de amenazas por el presidente Obama, que asumió la posibilidad de convivencia pacífica, conforme apuntó en entrevista a The Atlantic,

en la primera cumbre de las Américas a la que asistí, Hugo Chávez (...) continuaba siendo la figura dominante en la conversación (...). No nos gusta lo que está ocurriendo en Venezuela, pero no es una amenaza para los Estados Unidos (...). Y yo tuve que estar sentado allí y escuchar a [Daniel] Ortega (...) haciendo una diatriba de una hora contra los Estados Unidos. Pero estando allí, sin llevar todo eso a serio, porque en la realidad no era una amenaza para nosotros, ayudó a disminuir, a neutralizar el anti-americanismo en la región (Goldberg, 2016). 
La transformación operada entre su llegada a la presidencia en 2008 y la salida en 2016 de un cambio político favorable a Estados Unidos en las relaciones hemisféricas, pasa a ser leído por la administración Trump como oportunidad de ofensiva eliminadora del campo adversario.

El discurso de Bolton, así como el comunicado de la Casa Blanca de 30 de julio sobre Nicaragua, documentan ese abordaje. En el momento en que sectores organizados de oposición a gobiernos considerados hostiles consigan transformar situaciones de protesta callejera en movimiento destituyente que gane estatura de enfrentamiento violento de lado a lado, se explicita inmediatamente el apoyo estadounidense, con la Casa Blanca asumiendo como propia la caracterización de gesta "democratizadora", apostando en el cambio de régimen a través de sanciones y aislamiento internacional, conjuntamente con apoyo financiero que empodere la capacidad operativa opositora.

América Latina se torna palco experimental de abordaje funcional a nuevos tiempos de construcción de hegemonía, aplicable selectivamente en cualquier lugar del mundo en que se identifique oportunidad de alteración de correlaciones de poder favorable a los intereses del establishment dominante en la presidencia de Estados Unidos.

Con relación al estado de alerta frente a la presencia de Chinay Rusia en la región, existen niveles diferenciados de preocupación. En el caso chino, paralelamente a la ya mencionada ofensiva comercial de Trump en ámbito internacional, la postura es establecer convergencias con gobiernos aliados activando alarmas sobre un supuesto estilo de negocios predador por parte de la potencia asiática. Así lo expresa Mike Pompeo en entrevista al diario brasileño $O$ Estado de S. Paulo, después de participar de la toma de posesión del presidente Jair Bolsonaro.

Nosotros llegamos a una posición incuestionable de que China no puede ser liberada para envolverse en una actividad económica predatoria alrededor del mundo. Eso no es del interés de nadie. Donde China se presenta, en Brasil, Chile, Ecuador o cualquier parte, tiene que haber competición, transparencia y libertad (...) Eso no es apropiado y ustedes han visto que nuestro presidente [Trump] está preparado para luchar contra eso, dondequiera que, en cuestiones comerciales, América no encuentre prácticas justas y recíprocas de China. Eso vale para otras actividades en que China esté envuelta (Cantanhêde, 2019, traducción nuestra).

Si bien, como vimos, la tendencia en los años recientes es de una convergencia de gobiernos latinoamericanos favorable a Estados Unidos, esto no significa alineamiento automático con la agenda internacional del país. Es lo que ocurre en el caso de China, en que prevalecen intereses nacionales asociados a comercio exterior e inversiones. De acuerdo con estudio de la Comisión Económica para América Latina y Caribe (CEPAL), el país avanza sobre el segundo lugar en el 
intercambio comercial ocupado por la Unión Europea, con Estados Unidos manteniéndose en la primera posición. En términos de inversiones directas, en 2017 el stock de capital chino aumentó 46\% con relación a 2016, alcanzando 115 mil millones de dólares, tornándose el mayor inversionista externo en ese año. Argentina, Brasil y Perú se destacan como principales socios comerciales de China y receptores de $81 \%$ de la inversión directa entre 2005 y $2017 .{ }^{3}$ Con relación al financiamiento para el desarrollo en la región, la participación China correspondió a $80 \%$ de los préstamos entre 2005-2016, otorgados casi en su totalidad por bancos estatales, siendo que "la mayoría de los montos (93\%) fueron otorgados a la República Bolivariana de Venezuela (44\%), el Brasil (26\%), el Ecuador (12\%) y la Argentina (11\%)" (CEPAL, 2018, p. 22).

En el caso de Rusia, desde la llegada de Vladimir Putin al poder ha habido un creciente interés con la región. Estudio del Center for Strategic and International Studies (CSIS) destaca el caso de Venezuela, receptora de inversiones en la industria petrolera, principalmente a través de la empresa Rosneft, controlada mayoritariamente por el Estado, y destino de 75\% del total de armamentos exportados para América Latina (Miles, 2018). En el ámbito militar, las relaciones envuelven la cooperación entre las fuerzas armadas de los dos países, generando especulaciones en el gobierno estadounidense sobre ambiciones geopolíticas regionales rusas. Es el caso del arribo a Venezuela en diciembre de 2018 de delegación de oficiales para ejercicio conjunto, incluyendo dos aviones militares con capacidad de transportar misiles de largo alcance y armas nucleares. La reacción de Mike Pompeo, combinando la alerta de seguridad con críticas a los sistemas políticos de ambos países, tuvo tonos de Guerra Fría.

El gobierno de Rusia envió bombarderos del otro lado del mundo para Venezuela. El pueblo ruso y venezolano debía ver eso por lo que es: dos gobiernos corruptos derrochando fondos públicos y aplastando la libertad mientras su pueblo sufre (Arkhipov y Kravchenko, 2018, traducción nuestra).

\section{DISCURSO Y REALIDAD}

Más allá de la ofensiva retórica estadounidense sobre la importancia de la región, la disposición para generar cambios de gobierno en países no alineados y combatir la presencia china y rusa, los datos de la realidad muestran situación contradictoria con relación a recursos de poder disponibles y la decisión de utilizarlos en escala adecuada a los desafíos exaltados.

Diferentemente de China y Rusia, en que el Estado aparece como agente decisorio y ejecutor privilegiado de las políticas para la región, desplegando, especialmente en el caso chino, amplio stock de recursos para comercio, inversiones 
y financiamiento, en el caso estadounidense, decisiones de ese tipo corresponden esencialmente al sector privado, a partir de evaluaciones en que lógicas de mercado prevalecen sobre estigmas ideológicos. La actuación gubernamental tiene como protagonista destacado al Departamento de Estado, especialmente a través de la DA que, conforme apuntamos en la introducción, fue objeto de cortes por parte de la administración Trump, que valorizó las áreas de seguridad y defensa.

Del total mundial solicitado en el POE del Departamento de Estado para 2019, correspondiente a U\$S 40,2 mil millones, la parte para DA fue de 27,7 mil millones. ${ }^{4} \mathrm{Si}$ contemplamos específicamente el hemisferio occidental, la anunciada importancia de la región adquiere dimensiones reales. Conforme el cuadro 1, las solicitaciones de Obama para el año fiscal de 2017 son substancialmente mayores que las de Trump. En el caso de la DA y del Fondo de Apoyo Económico (ESF), que la administración actual reúne en una nueva área denominada Fondo para Apoyo Económico y Desarrollo (ESDF), hay reducción en la asignación presupuestaria si comparamos con la suma de lo contemplado para DA y ESF en 2017.

CUADRO 1

Presupuesto del Departamento de Estado para el hemisferio occidental (2017-2019) (En US\$ millones)

\begin{tabular}{|l|c|c|c|}
\hline & 2017 & 2018 & 2019 \\
\hline Hemisferio occidental & 1.739 .949 & 1.093 .551 & 1.110 .312 \\
\hline DA & 411.950 & - & - \\
\hline ESF & 513.374 & - & - \\
\hline ESDF & - & 516.081 & 515.949 \\
\hline
\end{tabular}

Fuente: United States of America (2016, p. 378; 2018b, p. 462).

Asociado a esos dos presupuestos, un dato a considerar sobre el status de la región en la diplomacia estadounidense es que en el momento de su elaboración aún no estaba definido el equipo del bureau de asuntos hemisféricos del Departamento de Estado, cabiendo la dirección al subsecretario interino Francisco Palmieri, oriundo de la administración Obama. El puesto será cubierto recién en octubre de 2018, cuando es nombrada Kimberly Breier, ex analista de la CIA, vinculada al think tank CSIS.

El nombramiento de Breier compone los dos principales cargos que tratan con la formulación de políticas regionales con funcionarios estrechamente afinados a los lineamientos del equipo Bolton-Pompeo. En septiembre, Mauricio Claver-Carone había sido indicado como asesor para el hemisferio occidental del Consejo de Seguridad Nacional. Con actuación anterior en el Departamento del Tesoro y en la directoria ejecutiva de Estados Unidos en el FMI, Claver-Carone exhibe notoria

4. Disponible en: <https://bit.ly/2KafAp8>. 
trayectoria militante contra los gobiernos de la llamada "troika de la tiranía", en que pesa también la dimensión personal como miembro de familia de exilados cubanos, además de sobrino de la primera esposa de Fidel Castro.

Frente al despliegue chino de una agenda de comercio, inversiones y financiamiento capaz de seducir gobiernos regionales en situación económica difícil, la ofensiva estadounidense carece de recursos de poder equivalentes para influenciar aliados a que compartan su ofensiva contra "actividades predatorias" de la potencia oriental.

La exacerbación activista contra "la troika de la tiranía”, amenazando países más débiles con un cerco de aislamiento dirigido a asfixiar y colapsar sus gobiernos, estaría aparentemente más próxima a la capacidad real de influencia de Estados Unidos. No obstante, la constitución de un apoyo considerable regional a esa postura se da fundamentalmente con relación a Venezuela, es menos vehemente para Nicaragua y de escaso apelo en el caso cubano.

El proceso de negociaciones para la normalización diplomática con Cuba llevado a cabo por Obama tuvo en cuenta presiones de gobiernos de signo político-ideológico diferente, tornadas cada vez más explícitas en las Cumbres de las Américas de Trinidad y Tobago en 2009, y Cartagena de Indias en 2012, concluyendo en el encuentro con Raul Castro en la reunión de Panamá de 2015 (Ayerbe, 2016). La cumbre de Lima de abril de 2018 no trajo novedades, su irrelevancia para Estados Unidos fue expuesta por la no participación de Donald Trump, primera vez en que el presidente del país propulsor de ese mecanismo diplomático, a partir de iniciativa de Bill Clinton en 1994, brilló por su ausencia. Obtener apoyo hemisférico para revivir cerco diplomático con retrovisor anticomunista no será tarea fácil.

En el ensayo monroísta trumpiano, contrapuntos entre discurso y realidad demarcan los alcances de las relaciones con América Latina. Retóricas inflamadas en tonos de Guerra Fría pretenden erigir enemigos y amenazas, sin la correspondiente disposición de recursos para el ejercicio de la política exterior, que se pretende compensar con la convocatoria de aliados dispuestos a asumir los costos de incierto protagonismo.

\section{REFERENCIAS}

ARKHIPOV, I.; KRAVCHENKO, S. Pompeo blasts Russia for strategic bombers sent to Venezuela. Bloomberg, 10 Dec. 2018. Disponível em: <https://bloom. $\mathrm{bg} / 2 \mathrm{SHCHMc}$.

AYERBE, L. F. Estados Unidos e América Latina: a construção da hegemonia. São Paulo: Editora Unesp, 2002. 
Estados Unidos y América Latina: balance de la administración Barack Obama al final de su segundo mandato. In: SERBIN, A. et al. (Org.). América Latina y el Caribe frente a la encrucijada actual de la globalización. Buenos Aires: CRIES, 2016. (Anuario de la integración regional de América Latina y el Caribe, n. 13).

BESHEER, M. Haley: Nicaragua risks becoming another Venezuela or Syria. VOA, 5 Sept. 2018. Disponível em: <https://bit.ly/2LFCOY4>.

BOLTON, J. Pay attention to Latin America and Africa before controversies erupt. The Hill, 1 Jan. 2018. Disponível em: <https://bit.ly/32SzwpP>.

CANTANHÊDE, E. "Ficamos satisfeitos com a oferta da base militar", diz Pompeo. O Estado de S. Paulo, 6 jan. 2019. Disponível em: <https://bit. ly/2VOVAhN>.

CEPAL - COMISIÓN ECONÓMICA PARA AMÉRICA LATINA Y EL CARIBE. Explorando nuevos espacios de cooperación entre América Latina y el Caribe y China. Santiago: CEPAL, 2018. Disponível em: <https://bit. ly/2K5ARRB $>$.

GOldBerG, J. The Obama doctrine. The Atlantic, Apr. 2016. Disponível em: <https://www.theatlantic.com/magazine/archive/2016/04/the-obamadoctrine/471525/>.

KERRY, J. Remarks on U. S. policy in the western hemisphere, 18 Nov. 2013. Disponível em: <https://2009-2017.state.gov/secretary/remarks/2013/11/217680.htm>.

MILES, R. Virtual Russian influence in Latin America. CSIS, 9 May 2018. Disponível em: <https://bit.ly/2Kb4bWX>.

PERRY, J. Após 2 meses de inquietação, a Nicarágua está numa encruzilhada fatídica. OPEU, 25 jul. 2018. Disponível em: <https://bit.ly/2K4jvok>.

THEATER, M. A. Remarks by president Trump on the policy of the United States towards Cuba. White House, 16 June 2017. Disponível em: <https://bit. ly/2YvnRxG>.

UNITED STATES OF AMERICA. Department of State. Fiscal Year 2017: foreign operations - appendix 3. 26 Feb. 2016. Disponível em: <https://2009-2017.state. gov/documents/organization/252733.pdf>.

. Office of Management and Budget. America first: a budget blueprint to make America great again. Washington: OMB, 2017. Disponível em: <https:// bit.ly/2nvjrBO>.

. Department of State. U.S. engagement in the western hemisphere.

1 Feb. 2018a. Disponível em: <https://www.state.gov/u-s-engagement-in-thewestern-hemisphere/>. 
Department of State. Fiscal Year 2019: foreign operations - appendix 2. 14 Mar. 2018b. Disponível em: <https://www.state.gov/wp-content/ uploads/2018/12/FY-2019-Department-of-State-Foreign-OperationsCongressional-Budget-Justification-Appendix-2-.pdf>.

Nacional Security Council. Remarks by National Security Advisor ambassador John R. Bolton on the administration's policies in Latin America. White House, 2 Nov. 2018c. Disponível em: <https://bit.ly/2HgC7BJ>.

. Office of Management and Budget. Efficient, effective, accountable: an American budget. Washington: OMB, 2018d. Disponível em: <https://bit. ly/2nTPE7s $>$. 